\title{
Neutron Diffraction Measurement of Residual Stresses in Al-clad U-10Mo Fuel Plates
}

D.W. Brown ${ }^{1 *}$, M. A. Okuniewski ${ }^{2,3}$, B. Clausen ${ }^{1}$, G.A. Moore ${ }^{3}$, T. A. Sisneros ${ }^{1}$

${ }^{1}$ Los Alamos National Laboratory, Los Alamos, New Mexico, 87545

${ }^{2}$ Purdue University, West Lafayette, IN 47907

${ }^{3}$ Idaho National Laboratory, Idaho Falls, Idaho 83415

*Corresponding Author

\begin{abstract}
Neutron diffraction was used to determine residual stress in monolithic two Al-clad U 10 weight percent Mo mini-fuel plates and a full sized fuel plate. One mini-plate was cooled following hot isostatic pressing at a rate of $6.75^{\circ} \mathrm{C} / \mathrm{min}$, the second at $0.675^{\circ} \mathrm{C} / \mathrm{min}$. A non-traditional method of calibrating the neutron diffractometer at each measurement point was necessitated by the thin nature of the sample. The in-plane stresses in the U-10Mo foils are relatively large, $-250 \mathrm{MPa}$ in the U-10Mo foil of the fast cooled mini-plate,-150MPa in the slow cooled mini-plate and -275 $\mathrm{MPa}$ in the full-sized plate. Likewise, the in-plane stresses in the Al-cladding of the fast-cooled mini-plate and full-sized plate were determined to reach $\sim 50 \mathrm{MPa}$, while in the slow-cooled sample the stresses in the Al cladding were on the level of the measurement uncertainty. The inplane stresses in the $\mathrm{Zr}$ diffusion barrier were estimated to be as large as $-300 \mathrm{MPa}$.
\end{abstract}




\section{Introduction}

In support of the United States' nonproliferation and highly enriched uranium (HEU) minimization policies, the U.S. Department of Energy (DOE)/National Nuclear Security Administration's (NNSA) Office of Materials Management and Minimization (M3) is actively working to convert civilian research and test reactors from the use of HEU fuel to low enriched uranium (LEU) fuel [1]. M3's Reactor Conversion program provides governments and facilities around the world with technical and economic assistance for conversion. If no suitable LEU fuels are available, the program contributes to the development of new LEU fuels. To date, M3 has converted or verified the shutdown of 87 research reactors worldwide, including 20 domestic facilities. Of the remaining domestic research reactors, five U.S. high performance research reactors and one associated critical assembly will require a new high density LEU fuel and fabrication capability, which is currently under development, to convert.

Existing qualified fuels do not meet the high fuel density requirements for the operation of these high-performance reactors, which include the Advanced Test Reactor (ATR) at Idaho National Laboratory, the High Flux Isotope Reactor (HFIR) at Oak Ridge National Laboratory, the University of Missouri Research Reactor (MURR), the Massachusetts Institute of Technology Reactor (MITR), and the Department of Commerce's National Bureau of Standards Reactor (NBSR). To maintain performance requirements, the Reactor Conversion program is developing a high density monolithic plate fuel system which uses low enriched uranium 10wt\% molybdenum (U-10Mo) foils clad with aluminum (Al-6061)[2]. A thin ( 0.025 mm) zirconium inter-diffusion barrier layer on either side of the U-10Mo foil prevents the formation of U-Al intermetallics, the properties of which may be detrimental to the fuel performance [1-3]. 
The thermal expansion of the aluminum cladding, zirconium diffusion barrier and U10 Mo fuel foil are roughly $\alpha_{\mathrm{Al}}=22 \times 10^{-6} / \mathrm{K}, \alpha_{\mathrm{Zr}}=5 \times 10^{-6} / \mathrm{K}$, and $\alpha_{\mathrm{U}-10 \mathrm{Mo}}=10 \times 10^{-6} / \mathrm{K}$, respectively. Note that the coefficient of thermal expansion of the zirconium is quoted as that in the a direction in the hexagonal close packed crystal structure because of the strong texture in the rolled zirconium foil, in which the a-axes are strongly preferred in the in-plane direction [4]. During cooling from the hot isostatic pressing (HIP'ing) temperature, the zirconium contracts considerably less than the U-10Mo, which in turn, contracts less than the aluminum. Because the aluminum dominates in terms of volume fraction, it is expected that the U-10Mo foil and zirconium bonding layer will be in a residual stress state of in-plane compression at room temperature after HIP'ing.

Smaller versions of full-sized fuel plates, "mini-plates", are often used for small scale experimentation and irradiation in test reactors. Measurements of the residual stress tensor in the U-10Mo foil in a mini-plate using high energy x-ray diffraction at the Advanced Photon Source (APS) [5] have been reported. However, determination of the stress state of the $\mathrm{Al}$ and $\mathrm{Zr}$ was not possible from this data because of "spottiness" in the diffraction pattern in each of these phases that results from poor grain sampling statistics. In general, increasing the divergence of the incident $\mathrm{x}$-ray beam can be used to solve the grain statistics issues better than increasing the sampling area, but that was beyond the scope of the original x-ray measurements. Moreover, current equipment at the APS does not allow for residual stress mapping in full size fuel plates, which are roughly six times larger than the mini-plates previously studied. Finally, x-rays are unlikely to afford the possibility of measurements in post-irradiated fuel because of the large $\gamma$ ray background emitted from the sample. Thus, it is desirable to develop and validate other residual stress measurement techniques. 
Neutron diffraction measurement of stress is well established [6,7] and avoids some of the issues inherent in synchrotron $\mathrm{x}$-ray measurements, although it cannot match the spatial resolution of high-energy x-ray measurements [5]. Sample positioning systems of modern engineering neutron diffractometers [8-11] routinely handle large samples and neutron beams have considerably larger divergence than synchrotron x-ray beams, alleviating the "spotty" diffraction issues by producing diffraction from more grains. Because neutrons penetrate into the bulk of materials, surface treatments, such as polishing and machining are typically not of import to residual stress measurements. Also, because all samples placed in the neutron beam are considered as activated upon removal, neutron facilities inherently have the infrastructure to handle radioactive samples. Finally, neutron detectors are relatively insensitive to $\gamma$-radiation emitted from samples, should stress measurements on irradiated fuels be desired. Measurements on irradiated fuel will create challenges, but these have been overcome, e.g. at the Chalk River neutron source through the intelligent use of shielding [12]. Neutron diffraction has not, however, been routinely used to determine stresses in thin foil samples, and creative methods of calibration must be utilized to accurately determine the stress field.

In this work, we have determined the residual stresses in the $\mathrm{Al}$ cladding, $\mathrm{Zr}$ diffusion barrier, and U-10Mo fuel foil in two mini-plates (one the same plate as [5]) following different thermal profiles and following a final dimensioning step using the Spectrometer for Materials Research at Temperature and Stress (SMARTS) [8] at the Lujan Neutron Scattering Center at Los Alamos National Laboratory. Residual stresses were also determined on a full-sized fuel plate, called the "OSU"-plate because it was destined for flow testing experiments at Oregon State University, which was over $660 \mathrm{~mm}$ long. The in-plane residual stresses, in particular in the 
aluminum cladding, are found to be large relative to the flow strength of the aluminum, which must be considered to be in an annealed state following the cladding procedure.

\section{Experimental Details}

\subsection{Sample Preparation}

The preparation of the mini-plates is discussed in detail elsewhere [2] and only a brief description will be given here. Depleted uranium and molybdenum feedstock were arc-melted in an inert atmosphere and cast into a $2.5 \mathrm{~mm}$ thick coupon and hot rolled at $923 \mathrm{~K}$. A zirconium diffusion barrier ( $\sim 0.015 \mathrm{~mm}$ thick) was co-rolled onto the fuel foil during the hot rolling process. The $\mathrm{Zr}$ was procured from Goodfellow as-annealed rolled 99.8\% pure Zr. Following hot-rolling, the foil was annealed at $923 \mathrm{~K}$ for 45 minutes followed by cold rolling to the final thickness of $\sim 0.28 \mathrm{~mm}$, nominally $0.25 \mathrm{~mm}$ of $\mathrm{U}-10 \mathrm{Mo}$ and two layers of $0.015 \mathrm{~mm}$ of the $\mathrm{Zr}$ interdiffusion prevention layer. The finished foils were hand polished with $15 \mu \mathrm{m}$ diamond paste and cleaned with ethanol before being bonded to the aluminum cladding (Al-6061 in the T6 condition). A recess which loosely fit the U-10Mo foil was machined into one side of the aluminum cladding, thus the cladding is thinner on one side of the mini-plate.

Six fuel plates were stacked vertically with graphite parting layers and tool steel strong backs separating each sample [2]. The samples were heated to the HIP'ing temperature of $833 \mathrm{~K}$ at $4.8 \mathrm{~K} / \mathrm{min}$ after which $104 \mathrm{MPa}$ of pressure was applied during the 90 minute soak time. One HIP run (designated run number 69) was cooled at a rate of $6.75^{\circ} \mathrm{K} / \mathrm{min}$ to room temperature, which is slightly faster than the typical HIP cooling regime for mini-plates[2]. A second HIP run (designated run number 68 ) was cooled at $0.675 \mathrm{~K} / \mathrm{min}$, the reasoning being that a scaled-up mass production of fuel plates would result in slower cooling times. One mini-fuel plate was 
removed from each HIP run, 69-4 (fast cooled) and 68-5 (slow cooled) for residual stress measurements. Following residual stresses measurements on the HIP'ed 69-4 mini-plate, it was returned to INL for shearing and thinning to final plate dimensions followed by repeat residual stress measurements. Mini-plate 69-4 in the HIP'ed condition is the same plate that was analyzed in the previously reported high-energy X-ray measurements [5].

A schematic of a mini-fuel plate is shown in figure 1a; locations of neutron measurements are indicated. The dotted line represents the boundary of the 69-4 plate following shearing to the final dimensions. The transverse (T) and longitudinal (L) directions of the sample are labeled in the figure and the sample normal $(\mathrm{N})$ direction is out of the page. The longitudinal direction of the sample is parallel to the rolling direction of the U-10Mo foil. The dimensions of the U-10Mo foil were $82 \mathrm{~mm}$ long, $19 \mathrm{~mm}$ wide and $0.28 \mathrm{~mm}$ thick $(0.25 \mathrm{~mm} \mathrm{U}-10 \mathrm{Mo}$ and $2 \mathrm{x}$ $0.015 \mathrm{~mm} \mathrm{Zr}$ ) and the final dimensions of the mini-plate were $150 \mathrm{~mm}$ x $50 \mathrm{~mm}$ x $1.61 \mathrm{~mm}$. Following shearing and thinning (symmetrically milled using fly cutter) to the final dimensions, the mini-plate was $101.4 \mathrm{~mm} \times 25.25 \mathrm{~mm} \times 1.39 \mathrm{~mm}$. The fuel foil maintained the same dimensions, but was not centered in the cladding. On one end (the end monitored), the edge of the fuel foil was only $4.7 \mathrm{~mm}$ from the edge of the plate, while on the other it was $18.7 \mathrm{~mm}$ from the edge of the plate. The buried edges of the U-10Mo foil were found by edge scans in the diffractometer. Throughout this report, the corner of the U-10Mo fuel foil will be used as the origin $(0,0)$.

The positions at which diffraction patterns were collected on the mini-plates are shown in figure 1a. Diffraction patterns were collected on the mini-plates along three line profiles parallel to the longitudinal axis of each sample; 1.) center line of the sample, $y=9.5 \mathrm{~mm}, 2$.) $2 \mathrm{~mm}$ from the edge of the U-10Mo foil, $y=2 \mathrm{~mm}$, and 3.) $2 \mathrm{~mm}$ off the edge of the fuel foil, $\mathrm{y}=-2 \mathrm{~mm}$. 
Obviously, some points had to be excluded following shearing to final dimensions. Also, long count time diffraction patterns were collected at the center of each mini-plate, $(x, y)=(41 \mathrm{~mm}$, $9.5 \mathrm{~mm}$ ), in order to determine the lattice parameters of the $\mathrm{Zr}$ diffusion barrier material at those positions.

The processing of the OSU plate followed the same path as the mini-plates as closely as possible. The OSU plates were sealed in stainless steel can and heated to the HIP'ing temperature of $833 \mathrm{~K}$ at $4.8^{\circ} \mathrm{C} / \mathrm{min}$ after which $104 \mathrm{MPa}$ of pressure was applied during the 90 minute soak time. Subsequently, the HIP assembly was cooled at $\sim 6.7 \mathrm{~K} / \mathrm{min}$ to room temperature. A schematic of the OSU plate is shown in figure $1 \mathrm{~b}$; locations of neutron measurements are indicated. The dimensions of the OSU plate were $660.5 \mathrm{~mm}$ longitudinal, $127.4 \mathrm{~mm}$ transverse, and $2.03 \mathrm{~mm}$ thick; the enclosed $\mathrm{U}-10 \mathrm{Mo}$ foil was $580 \mathrm{~mm}$ long, $88.9 \mathrm{~mm}$ wide, and nominally $0.25 \mathrm{~mm}$ thick. The $\mathrm{Zr}$ diffusion barrier was $0.015 \mathrm{~mm}$ thick on each side of the U-10Mo.

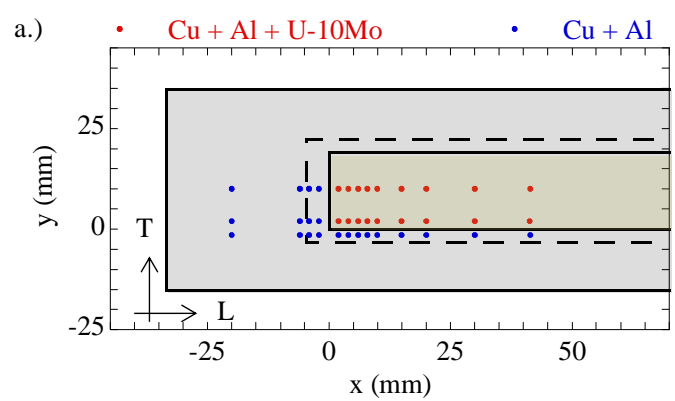

b.)

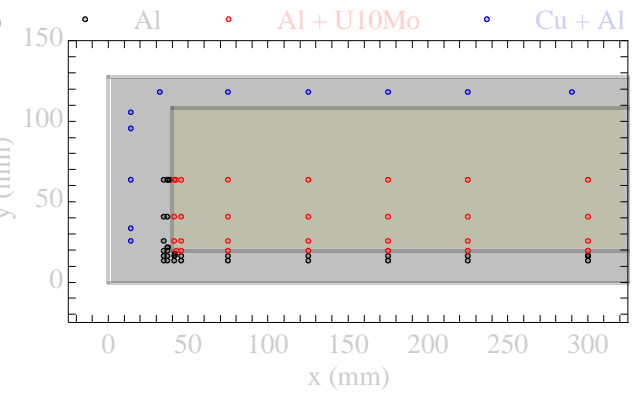

Figure 1. Schematic of the (a.) mini-plate and (b.) OSU samples with an overlaid plot of the measurement positions. The origin of the axis used throughout this report is as shown. The longitudinal (2) and transverse (1) directions are indicated. The normal (3) direction is out of the page.

\subsection{Residual Strain/Stress Measurement}

The residual stress measurements were completed on the SMARTS diffractometer at Los Alamos Neutron Science Center (LANSCE) at Los Alamos National Laboratory. SMARTS accepts a white neutron beam (continuous wavelength spectrum from $0.5 \AA-4.0 \AA$ ) from a $283 \mathrm{~K}$ water moderator located roughly $31 \mathrm{~m}$ from the sample position. A schematic of the diffraction geometry is 
shown in figure $2 \mathrm{a}$ and pictures of the samples in position in fig. $2 \mathrm{~b}$ (mini-plates) and $2 \mathrm{c}$ (OSU sample). The incident neutron beam was defined using computer controlled motorized boronnitride slits (set to $2 \times 5 \mathrm{~mm}$ ) and impinged on the sample. The slits were defined to optimize the spatial resolution in the scanning (longitudinal) direction, e.g. when the sample longitudinal direction was vertical, as in figure $2 b$, the vertical slit was $2 \mathrm{~mm}$.

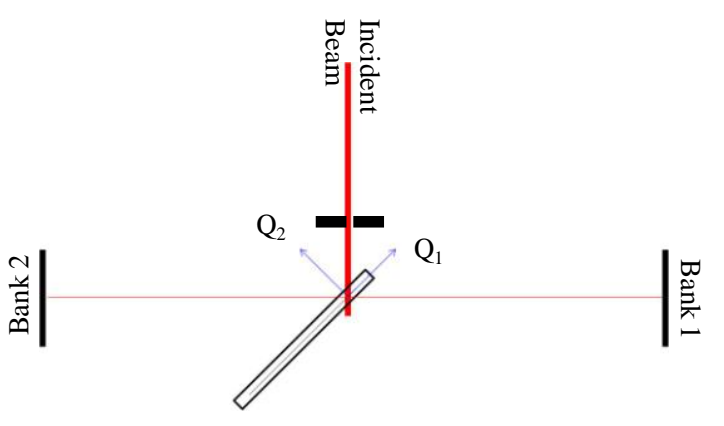

a.)

b.)

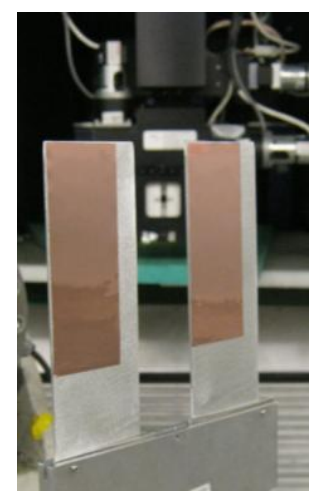

c.)

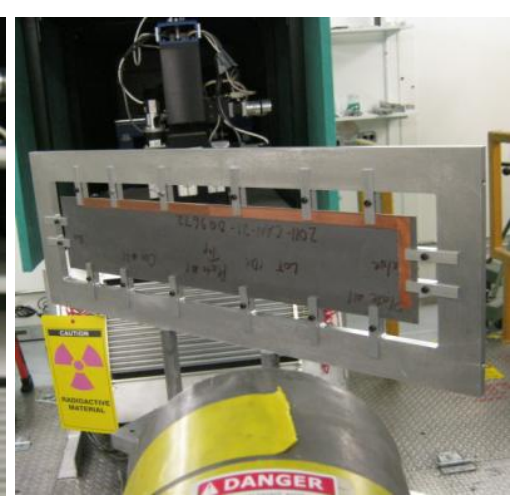

Figure 2. a.) Schematic of the diffraction geometry of SMARTS indicating the diffraction vectors of each detector bank. Pictures of the b.) the mini plates and c.) OSU plate in SMARTS. The samples are shown as mounted to determine the lattice parameters in the $\mathrm{T}$ and $\mathrm{N}$ sample directions of the mini plates and $\mathrm{N}$ and $\mathrm{L}$ sample directions of the OSU plate in banks 1 and 2 , respectively.

The neutrons are scattered from the illuminated portion of the sample, called the "gauge volume", and collected in two fixed angle detector banks (called banks 1 and 2) situated at $\pm 90^{\circ}$ from the incident beam, each defining a unique diffraction vector $(\mathrm{Q})$ at $\pm 135^{\circ}$ from the incident beam. The samples were mounted on a computer controlled translator table [8] with one corner of each sample located with respect to the known center of the diffractometer to better than $\pm 0.1 \mathrm{~mm}$ accuracy using computerized theodolites. The samples are oriented such that an in-plane direction of the sample is parallel to one diffraction vector, and the sample normal direction is parallel to the other.

In Figure 2b, mini plates 69-4 and 68-5 are shown mounted for measurement of lattice parameters normal to the plate surface in bank 2 and parallel to the transverse direction in bank 
1. The sample was moved with respect to the gauge volume and diffraction patterns were collected at selected positions on the mini-plates, indicated in figure 1a. Following this set of measurements, the mini-plates were re-oriented to determine the normal direction lattice parameters in bank 1 and the longitudinal lattice parameters in bank 2 . The measurement of the normal direction lattice parameters in the opposing detector banks provides a strong test of the instrument calibration procedure.

Figure 2c shows the OSU plate mounted in SMARTS for the measurement of the normal (longitudinal) lattice parameter in bank 1 (2). Chronologically, the OSU plate measurement was completed first. It was realized after the measurement of the residual stresses in the OSU plate that validation of the calibration could be attained for free, so to speak, by measuring the $\mathrm{N}$ lattice parameter independently in the two banks. In the case of the OSU plate, the $\mathrm{N}$ lattice parameter was determined twice in bank 1; the $\mathrm{L}$ and $\mathrm{T}$ lattice parameters in bank 2.

In typical neutron diffraction measurements of residual stress on a white beam source, the detector view is focused using radial collimators [6, 13-15] defining the gauge volume in the third dimension. This practice both provides depth resolution as well as ensures that the gauge volume does not move with respect to the diffractometer during profiling resulting in large fictitious strain measurements [16]. However, due to the thin foil nature of these samples, that practice was not possible and diffraction data was collected from the entire thickness of the samples. Thus, there is no through-thickness resolution in these measurements. The samples studied herein are very thin relative to their in-plane directions and little through-thickness variation of stresses is expected. However, the collected diffraction patterns represent an average stress measurement through the thickness of the sample for each of the phases. 
Further, because the gauge volume is not defined in three dimensions, small shifts of the samples from the diffractometer center with the motion of the translator table, which are almost inevitable because the samples were not perfectly straight, will result in fictitious strains. This effect can be large compared to mechanical strains [16] and is generally avoided at all cost. This was anticipated, and an on-board calibrant in the form of an annealed thin copper foil $(0.07 \mathrm{~mm})$ was attached symmetrically to each face of the samples to detect and correct the fictitious strains. Annealed copper was chosen because the diffraction peaks from copper do not interfere with those of $\mathrm{Al}, \mathrm{U} 10 \mathrm{Mo}$, or $\mathrm{Zr}$ and because, in the annealed state, the strength of the copper is very low limiting the potential residual stresses in the "calibrant", thus limiting the possible errors from assuming it is stress free.

While this procedure provides a good calibration of the two detector banks against each other and between the several samples probed (to be demonstrated below), it should not be considered an absolute calibration. Absolute calibration, to the accuracy required for lattice strain measurement, is extremely difficult to achieve. Because the beam penetrates into the bulk of the sample, the calibrant would not only have to be verified stress free, such as a NIST standard powder, but would also have to have the same geometry as well as neutron scattering and absorption cross section as the sample. Otherwise, the "center of diffraction" is effectively shifted because scattering from the gauge volume is not evenly distributed, but weighted by both absorption and scattering strength. This again results in fictitious strains. Validation of the calibration was obtained by rotating the sample $90^{\circ}$ about a vertical axis when re-orienting from the transverse/normal (1/3) lattice parameter measurement to the longitudinal/normal (2/3). This results in the two normal lattice parameter determinations occurring in the opposing banks and will provide a strong validation of the calibration procedure for the mini-plate measurements. 
The current method of using an on-board calibrant symmetrically placed on each side of the sample allows us to accurately compare lattice parameters determined along multiple sample directions, in this case, the N, L, and T directions. The difference in lattice parameter in the three directions is directly related to the deviatoric strain and stress tensors but, without further assumption, is insensitive to an arbitrary hydrostatic stress. However, due to the thinness of the sample, it is reasonable to assume that the out-of-plane stress components are zero far from any boundaries, i.e. at the center of the sample. The maximum out-of-plane stress at the center of the fuel plate in the aluminum can be estimated based on the dimensions and elastic properties of 6061 aluminum in the annealed state [17].

A point of interest concerning this method is that spatial variation in solute chemistry becomes irrelevant because the effect of chemistry on lattice parameter is independent of sample direction. Thus, the deviatoric stress component at a minimum can be determined without detailed knowledge of chemistry variations in the sample. This is an important consideration for measuring residual stresses in post-irradiated fuel plates because the burn-up (and thus the solute chemistry) is a function of position and may not be well known.

\subsection{Data Analysis}

Whole pattern Rietveld refinements of the diffraction patterns were accomplished using the GSAS software developed at LANL [18] using TOF profile function 1 [19]. The data was analyzed to determine the crystallographic texture, lattice parameters, and peak variances of each phase.

2.3.1Texture Time-of-Flight (TOF) neutron diffraction is ideal for determining a specific projection of the orientation distribution function, that is the inverse pole figure (IPF) [20]. The 
IPF is a plot of pole density as a function of crystal orientation (hkl) along a specific sample direction, e.g. the $\mathrm{N}$ direction, on an irreducible sterograph. This is precisely what is measured on a TOF diffractometer with fixed detector banks, that is with the diffraction vector aligned with a specified sample direction, e.g. the $\mathrm{N}$ direction. The pole densities are determined with GSAS using a spherical harmonic expansion of the orientation distribution function [21]. The coefficients of the spherical harmonics are determined by fitting the observed peak intensities to those calculated. The multiplicative correction factors for the preferred orientation in the Rietveld refinement are the Multiples of Random Distribution (MRD) plotted on the irreducible stereograph to produce IPF's [22].

2.3.2 Residual Stress The lattice parameter, $a$, was determined at each measurement position $(x, y)$ and the elastic lattice strain was determined from $\varepsilon(x, y)=\frac{a(x, y)-a_{0}}{a_{0}}$ (eq.1), where $a_{0}$ represents a reference, stress-free, lattice parameter. The uncertainty in the lattice parameter measurement, $\sim 0.0001 \AA$ (relative uncertainty, corresponding to a strain uncertainty of $\sim 0.00003$ or $30 \mu \varepsilon$ ), is determined from the estimated standard deviation in the fit to the lattice parameter and, thus, only represents statistical uncertainty and ignores systematic uncertainty which may result, for instance, from misalignment of the sample or assumptions of the reference lattice parameter.

Acceptable reference samples were not available for the aluminum or the U-10Mo because it was not possible to fabricate a reference sample without sending it through the identical thermo-mechanical procedures. For instance, it is known that the Mo content changes during the HIP'ing procedure as $\alpha-U$ precipitates $[4,5]$. 
The reference lattice parameters of the U-10Mo and $\mathrm{Al}$ were determined by forcing the normal component of the stress $\left(\sigma_{33}\right)$ to zero at the center of the plate, far from any boundary. Simple beam bending theory [23] can be used to estimate the order of magnitude of the maximum out of plane stress at the center of the fuel plate given the dimensions and elastic properties of $6061 \mathrm{Al}$ in the annealed state. Assuming a uniform load perpendicular to a beam that is simply supported at both ends, the maximum bending moment at the center of the beam is $M_{\max }=\frac{q L^{2}}{8}$ where $\mathrm{q}$ is the distributed load and $\mathrm{L}$ is the beam length. The maximum longitudinal stress at the surface of the beam is $\sigma_{x}(y)=\frac{M}{I} y$, where $I=\frac{b h^{3}}{12}$ is the moment of inertia for the beam and y is half the beam height, $\frac{h}{2}$, assuming that there are no normal stresses in the beam. We assume that the maximum normal stress achievable in the foil is the yield stress of the Al, and that the beam is $1 \mathrm{~mm}$ wide, $1 \mathrm{~mm}$ tall and $20 \mathrm{~mm}$ long, approximating the aspect ratio of the cross-section of the fuel plate. Then we can establish the ratio between the applied distributed load, which is representing the out-of-plane stress, and the yield stress to be $\frac{\sigma^{Y}}{q_{\max }}=\frac{3}{4} \frac{L^{2}}{b h^{2}}=300$ . Hence we find that the maximum value of the out-of-plane stress is 300 times less than the yield stress, or well less than $1 \mathrm{MPa}$.

The residual strains are determined in three orthogonal directions, $\mathrm{N}, \mathrm{L}$, and $\mathrm{T}$. The residual stresses in the same directions are then determined from Hooke's law : $\sigma_{L}=\frac{E}{1+v}\left\{\varepsilon_{L}+\left[\frac{v}{1-2 v}\left(\varepsilon_{L}+\varepsilon_{T}+\varepsilon_{N}\right)\right]\right\}$ (eq.2) [24] 
where we have used $\mathrm{E}=70 \mathrm{GPa}$ and $v=0.33$ for the aluminum and $\mathrm{E}=86 \mathrm{GPa}$ and $v=$ 0.35 for U-10Mo [25-27]. The indices can be adjusted as necessary to solve for the remaining stress components.

Determining residual stresses in the $0.015 \mathrm{~mm}$ thick $\mathrm{Zr}$ diffusion prevention layer is more difficult. The crystallographic texture of the $\mathrm{Zr}$ diffusion barrier is such that only the c lattice parameter can be determined with sufficient accuracy in the $\mathrm{N}$ direction and the a lattice parameter in the $\mathrm{L}$ and $\mathrm{T}$ directions. Thus, the lattice parameters cannot simply be compared in multiple sample directions as done for the $\mathrm{U}-10 \mathrm{Mo}$ and the $\mathrm{Al}$ to find the deviatoric stress components. However, the $c_{N} / a_{L}$ and $c_{N} / a_{T}$ ratios can be calculated with a great deal of accuracy $( \pm 0.0005)$. Taking the ratio of the lattice parameters removes issues with absolute calibration, and the experimentally determined ratio can be compared to that found in literature for pure $\mathrm{Zr}$ and that observed for the as-received $\mathrm{Zr}$ (prior to roll-bonding) to get an indication of the stress state of the diffusion barrier.

Because the $\mathbf{c}$ and $\mathbf{a}$ axes are normal to each other, the specified ratios characterize two distinct grain sets that actually exist in the diffusion barrier, that is 1.) $\mathbf{c} \| \mathrm{N}$ and all or 2.) $\mathbf{c} \| \mathrm{N}$ and allT. Moreover, because these orientations represent the dominant grain orientation in the $\mathrm{Zr}$, thermal residual stresses due to the anisotropic thermal expansion of $\mathrm{Zr}$ should be minimal in these grains $[28,29]$.

While a quantitative determination of the residual stresses in the $\mathrm{Zr}$ layer will not be possible from the current data, the stresses in the barrier can be estimated as follows. We approximate the stress state of the $\mathrm{Zr}$ diffusion prevention layer as in-plane equi-biaxial, that is 
$\sigma_{N}=0$ and $\sigma_{L}=\sigma_{T}$, resulting in the simplification $\varepsilon_{L}=\varepsilon_{T}=\frac{1-v}{E} \sigma_{L}$ (eq. 3) and $\varepsilon_{N}=\frac{-2 v}{E} \sigma_{L}$ (eq. 4). In the dominant grain orientation, i.e. c-axis parallel to $\mathrm{N}$ and a-axes parallel to $\mathrm{L}$ (or T), the strain components can be written as $\varepsilon_{N}=\frac{c-c_{0}}{c_{0}}$ or $c=c_{0}\left(1+\varepsilon_{N}\right)$, where $\mathrm{c}_{0}$ is the reference lattice parameter and $\mathrm{c}$ the lattice parameter under conditions of stress. The same relation can be written between the a lattice parameter and the $\mathrm{L}$ and $\mathrm{T}$ strains. The $\frac{c_{N}}{a_{L}}$ ratio at stress can then be written $\frac{c_{N}}{a_{L}}=\frac{c_{0}\left(1+\varepsilon_{N}\right)}{a_{0}\left(1+\varepsilon_{L}\right)}$ (eq 5). Substituting equations 3 and 4 into equation 5 and solving for $\sigma_{L}$, the longitudinal stress component can be estimated as $\sigma_{L}=\frac{E\left(1-\frac{c_{N} / a_{L}}{c_{0} / a_{0}}\right)}{(1-v)\left(\frac{c_{N} / a_{L}}{c_{0} / a_{0}}+2 v\right)}($ eq 6).

Using a representative literature value of $\frac{c_{0}}{a_{0}}=1.5928$ [30-32], and appropriate elastic properties for $\mathrm{Zr}(\mathrm{E}=100 \mathrm{GPa}$ and $\mathrm{n}=0.3$ [33]) the in-plane component of the residual stress can be estimated from eq. 6. Note, this estimation assumes isotropic elastic properties of $\mathrm{Zr}$, which is not a good approximation and the assumption of equi-biaxial stress is also suspect, but they are sufficient to get an estimate of the residual stress in the diffusion prevention layer.

\section{Results}

\subsection{Texture}




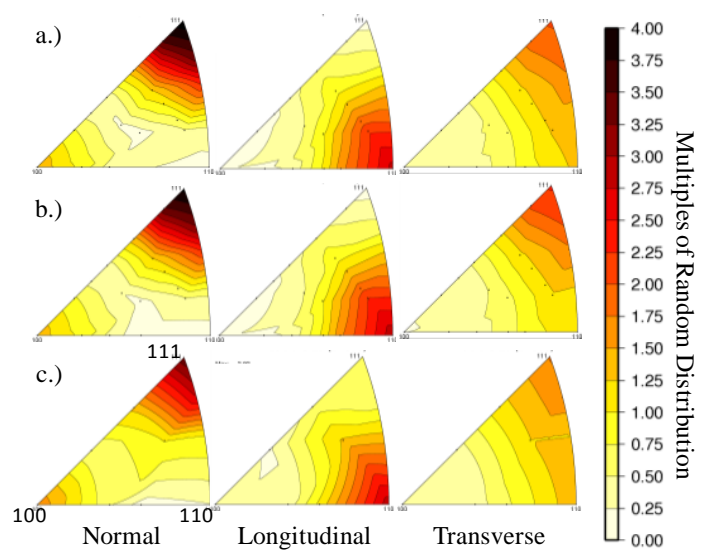

Figure 3. Inverse pole figures representing the texture of the U10 Mo in the (a.) fast cooled (69-4), (b.) slow cooled (68-5) and (c.) OSU plates. Contours go from 0 to 4 multiples of random distribution (MRD) at 0.25 intervals.
Inverse pole figures representing the crystallographic texture of the U-10Mo fuel foil in mini-plate samples 69-4 and 68-5 and the OSU plate are shown in figures $3 \mathrm{a}-\mathrm{c}$, respectively. The textures of the two mini-plates are equal within our uncertainty, that of the OSU plate is slightly weaker. The texture is moderately strong, $\sim 4$ multiples of random distribution (MRD), and typical of a body-centered cubic rolling texture.
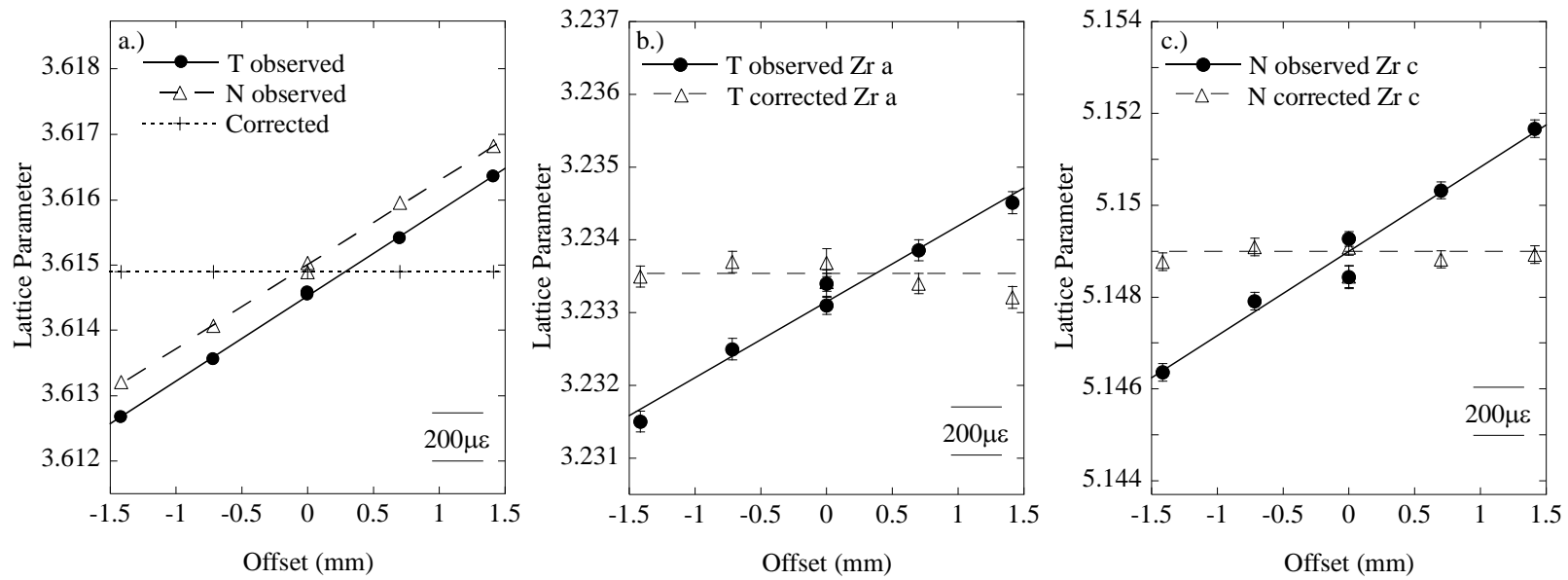

Figure 4. a.) Raw and corrected $\mathrm{Cu}$ and $\mathrm{Zr}$ b.) transverse and c.) normal lattice parameters as a function of distance offset from the diffractometer center.

That is, the (110) plane normals are parallel to the rolling (longitudinal) direction.

\subsection{Calibration}

To test the robustness of calibration measurement, diffraction patterns were collected on the $\mathrm{Zr}$ reference foil (as received from Goodfellow) with the on-board $\mathrm{Cu}$ calibrant as a function of displacement from the diffractometer center parallel to the incident beam direction. The foil 
$\mathrm{N}$ direction was aligned with the bank 1 diffraction vector, the $\mathrm{T}$ direction with bank 2 . Figures 4a-c show the observed (raw) and corrected a.) Cu lattice parameters in both directions, b.) $\mathrm{Zr}$ lattice parameter a in the $\mathrm{T}$ direction and $\mathrm{c}$.) $\mathrm{Zr}$ lattice parameter $\mathrm{c}$ in the $\mathrm{N}$ direction. The scale of each plot is such that full scale represents a strain of roughly $2000 \mu \varepsilon$ : a span of $200 \mu \varepsilon$ is indicated in each plot. Large fictitious strains are evident in each unique lattice parameter as the sample is displaced from the diffractometer center, consistent with previous calculations [16]. A multiplicative constant was determined to correct the observed copper lattice parameter to its reference value, 3.6149 [34] and applied to the Zr lattice parameters. Once corrected by the calibration procedure, the $\mathrm{Zr}$ lattice parameters are independent of displacement to within the uncertainty, validating the use of the on-board calibrant to correct for small sample displacement. The standard deviation of the corrected a and $\mathbf{c}$ values are $0.00019 \AA$ and $0.00024 \AA$, respectively, corresponding to "strains" of $\sim 0.00005$ or $50 \mu \varepsilon$. It is reasonable to take this as the uncertainty induced by the unique calibration procedure. This is critical for this study because while the mini-plate 69-4 was quite flat, mini-plate 68-5 was noticeably curved and precise alignment would have been difficult or impossible to achieve.

We note that within the measurement uncertainty, the $\frac{c_{N}}{a_{T}}$ ratio determined in the reference $\mathrm{Zr}$ foil is independent of the offset from the diffractometer center and was determined to be $1.5925 \pm 0.0005$, consistent with the published ratio [30-32]. 

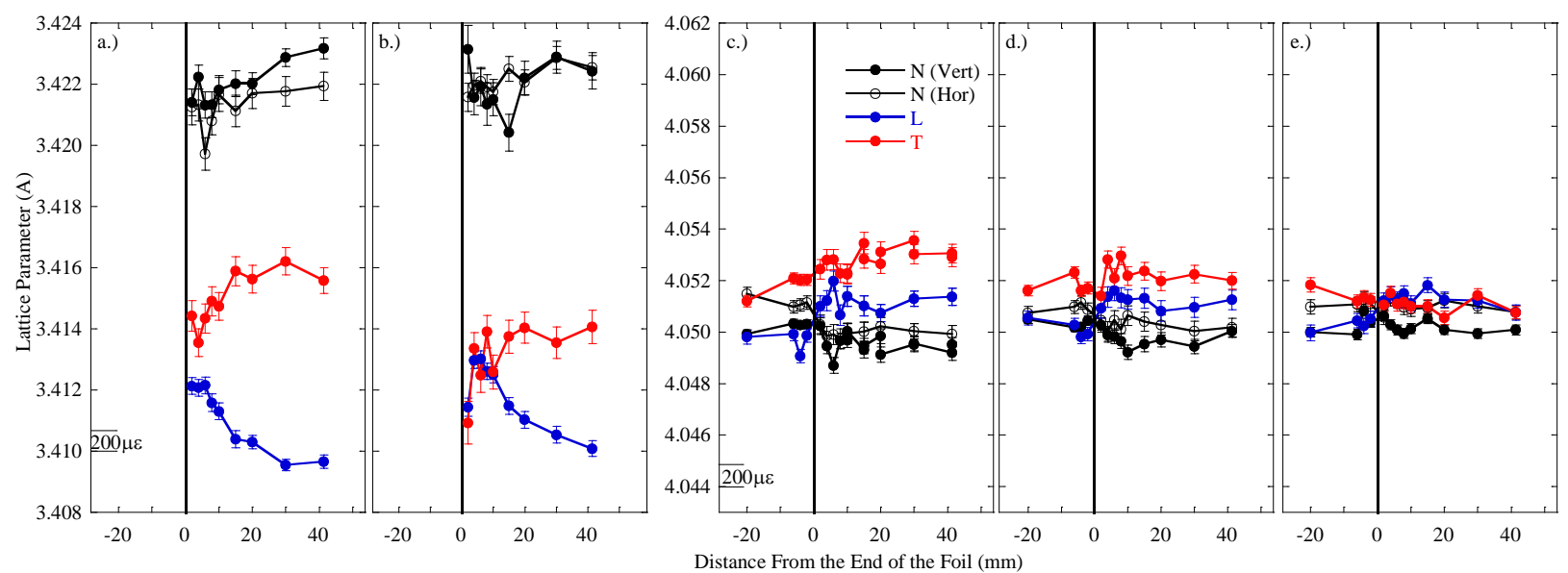

Figure 5 a,b.) U10Mo lattice parameters measured in the three sample directions in the fast cooled sample, $69-4$, as a function of distance from the end of the foil on the a.) center line and b.) $2 \mathrm{~mm}$ from the edge of the fuel foil. c-e.) Same for Al lattice parameters measured on the c.) center line, d.) $2 \mathrm{~mm}$ from the edge of the fuel foil, and e.) $2 \mathrm{~mm}$ off the edge of the fuel foil. The open and closed black symbols represent the two independent measurements of the normal lattice parameter with the sample vertical and horizontal, respectively. The vertical black line at $0 \mathrm{~mm}$ represents the edge of the fuel foil.

\subsection{Lattice Parameters}

Figures 5a and b show the U-10Mo lattice parameter measured in the sample normal, longitudinal and transverse directions as a function of position on the two line profiles on the U10Mo foil in sample 69-4, cooled at $6.75 \mathrm{~K} / \mathrm{min}$ ("fast" cooled). Figures 5c-e show the Al lattice parameters measured on the three line profiles. The vertical black line indicates the edge of the U-10Mo foil on each plot. The scales of the abscissa in the U-10Mo and Al plots are such that the full range represents roughly the same strain, i.e. $5000 \mu \varepsilon$.

The two independent measurements of the lattice parameters in the normal direction are shown as solid (sample vertical) and open (sample horizontal) symbols. The agreement between the two measurements in the opposing detector banks is very good, near the level of statistical uncertainty. This argues convincingly that the use of the on-board copper calibrant has successfully calibrated the two banks against each other allowing for direct comparison of lattice parameters determined in the two banks. 
The dependence of the lattice parameter on sample direction in the U-10Mo in the fast cooled sample is large, especially when compared to the difference between the two independent measurements of the lattice parameter in the $\mathrm{N}$ direction. On both line profiles (center and edge of the foil), the U-10Mo lattice parameter in the $\mathrm{N}$ direction is considerably larger than either the $\mathrm{T}$ or $\mathrm{L}$ directions; the $\mathrm{T}$ lattice parameter is somewhat larger than the $\mathrm{L}$ lattice parameter. On both lines, the L lattice parameter tends to decrease with distance from the edge of the U-10Mo foil, while the $\mathrm{T}$ lattice parameter increases. The variation of the U-10Mo lattice parameter determined in the sample $\mathrm{N}$ direction is on the level of the statistical uncertainty.

Compared to the observations in the $\mathrm{U}-10 \mathrm{Mo}$, there is much less directional dependence of the $\mathrm{Al}$ lattice parameter (in a relative sense) on the center and edge line profiles, and the trend is reversed with respect to the in-plane and out-of-plane lattice parameters. The Al lattice parameters in the in-plane ( $\mathrm{L}$ and $\mathrm{T}$ ) sample directions are larger than that in the $\mathrm{N}$ direction, with the T lattice parameter being consistently the largest. On the line profile that is off of the U10Mo foil, the direction dependence of the Al lattice parameter is near the level of the measurement uncertainty. 


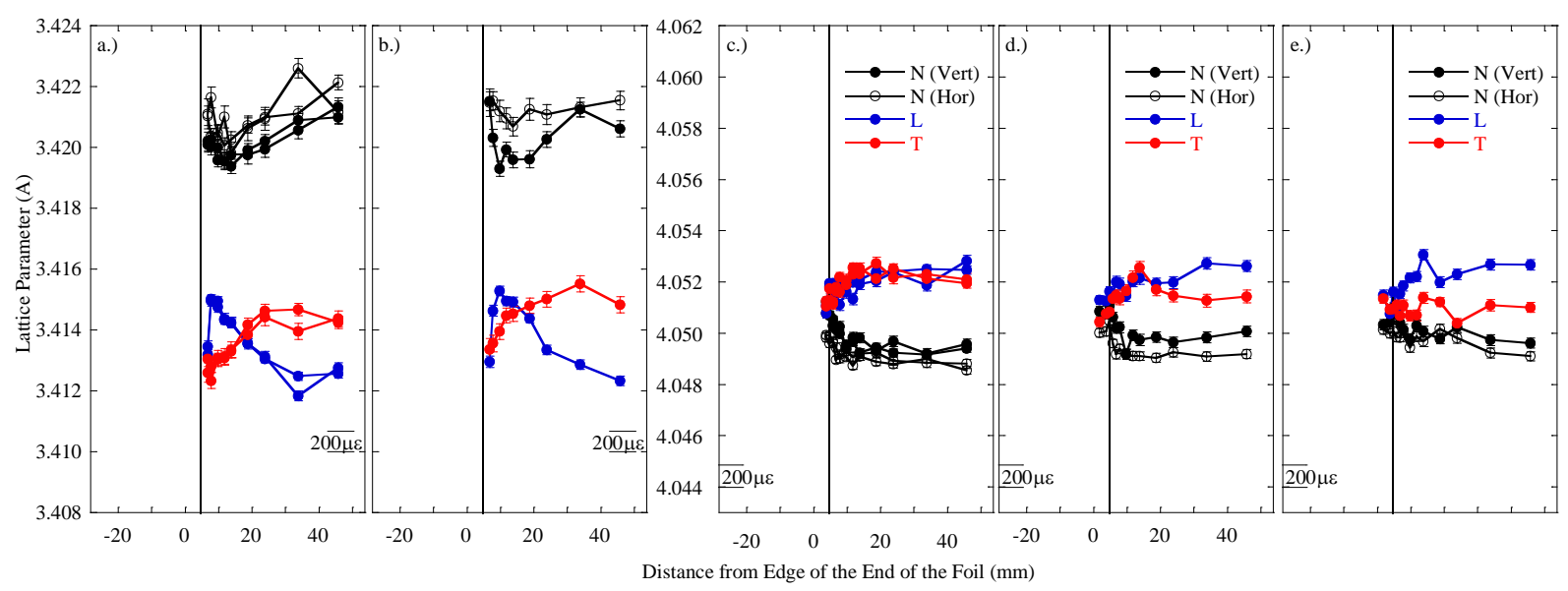

Figure 6 a,b.) U10Mo lattice parameters measured in the three sample directions in the fast cooled sample, 69-4, following shearing and thinning to final dimensions as a function of distance from the end of the foil on the a.) center line and b.) $2 \mathrm{~mm}$ from the edge of the fuel foil. c-e.) Same for Al lattice parameters measured on the c.) center line, d.) $2 \mathrm{~mm}$ from the edge of the fuel foil, and e.) $2 \mathrm{~mm}$ off the edge of the fuel foil.

Figures 6a-e show similar plots (same scales) of the lattice parameter determined on the same mini-plate (69-4) following shearing and thinning of the Al cladding to final dimensions. The line profile at the center of the sample was measured twice. There is an obvious change in lattice parameter in each direction, both absolute and relative to the other directions, following shearing. The $\mathrm{N}$ lattice parameter decreases slightly. The difference between two in-plane lattice parameters ( $\mathrm{L}$ and $\mathrm{T}$ ) for the $\mathrm{U}-10 \mathrm{Mo}$ decreases. In the cladding, the separation between the $\mathrm{Al}$ lattice parameters increases following shearing and thinning. The in-plane Al lattice parameters are larger than the $\mathrm{N}$ lattice parameter, in particular near the center of the plates. The separation trends to zero near the edge of the fuel foil and once off the foil.

As stated earlier, the reference lattice parameters, $\mathrm{a}_{0}$, for both the U-10Mo and Al have been determined by forcing the $\mathrm{N}$ component of the stress to zero near the center of the plates. It is interesting to examine the variation of $\mathrm{a}_{0}$ between the two measurements of mini-plate 69-4, before and after shearing because that should not change. For both the U-10Mo foil and Al cladding, the observed reference lattice parameters were roughly $60 \mu \varepsilon$ less in the sheared plate 
than the as HIP'ed plate, speaking strongly of the repeatability of the measurements, despite being separated by months.
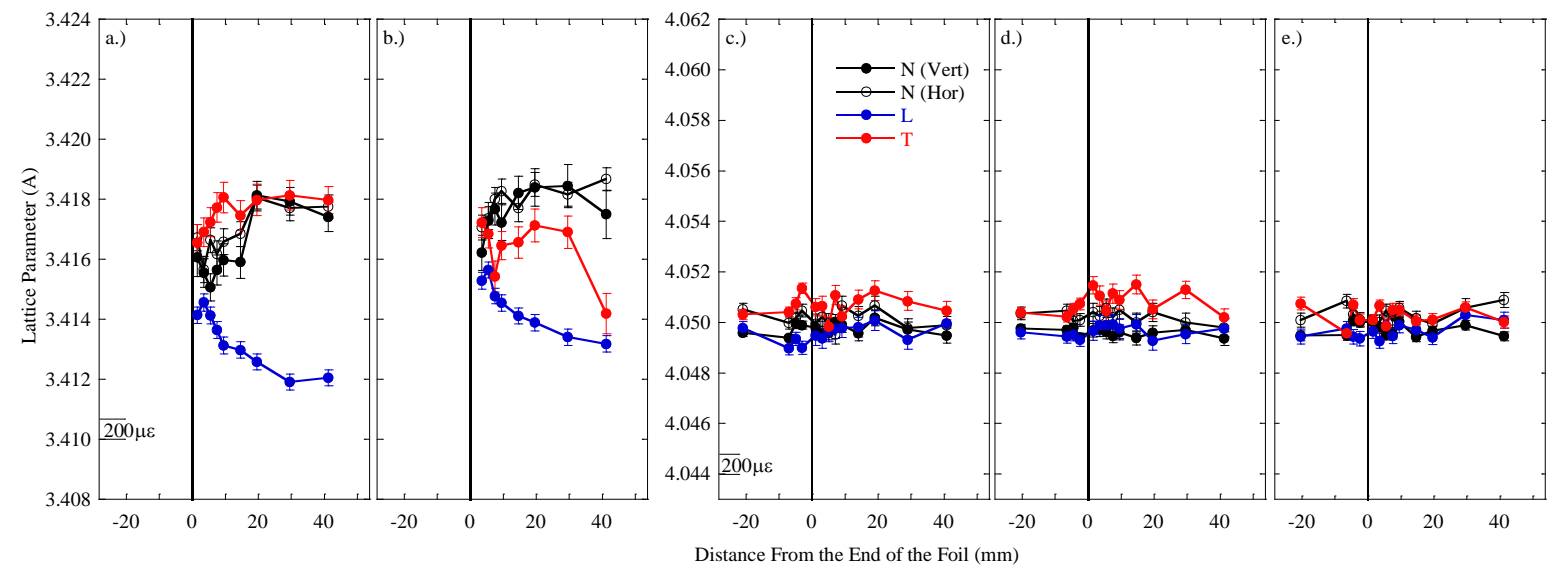

Figure 7 a,b.) U10Mo lattice parameters measured in the three sample directions in the slow cooled sample, 68-5, as a function of distance from the end of the foil on the a.) center line and b.) $2 \mathrm{~mm}$ from the edge of the fuel foil. c-e.) Same for Al lattice parameters measured on the c.) center line, d.) $2 \mathrm{~mm}$ from the edge of the fuel foil, and e.) $2 \mathrm{~mm}$ off the edge of the fuel foil.

Figures 7a-e show similar plots (same scales) of the lattice parameter determined on sample $68-5$, cooled at $0.675^{\circ} \mathrm{C} / \mathrm{min}$. The in-plane lattice parameters exhibit a similar separation as in the 69-4 mini-fuel plate; the transverse lattice parameter is consistently somewhat larger than the longitudinal. However, the lattice parameters in both of the in-plane directions have increased, and that in the normal direction decreased such that the normal and transverse lattice parameters are nearly within uncertainty of each other. Similarly, the difference in the Al lattice parameter observed in different sample directions is reduced to roughly the level of the uncertainty in the slow cooled sample.

It is not expected that the reference lattice parameters of the U-10Mo foil and Al cladding would agree between mini-plates $69-4$ and 68-5 because the thermal profiles were very different, which likely results in different solute chemistry. 

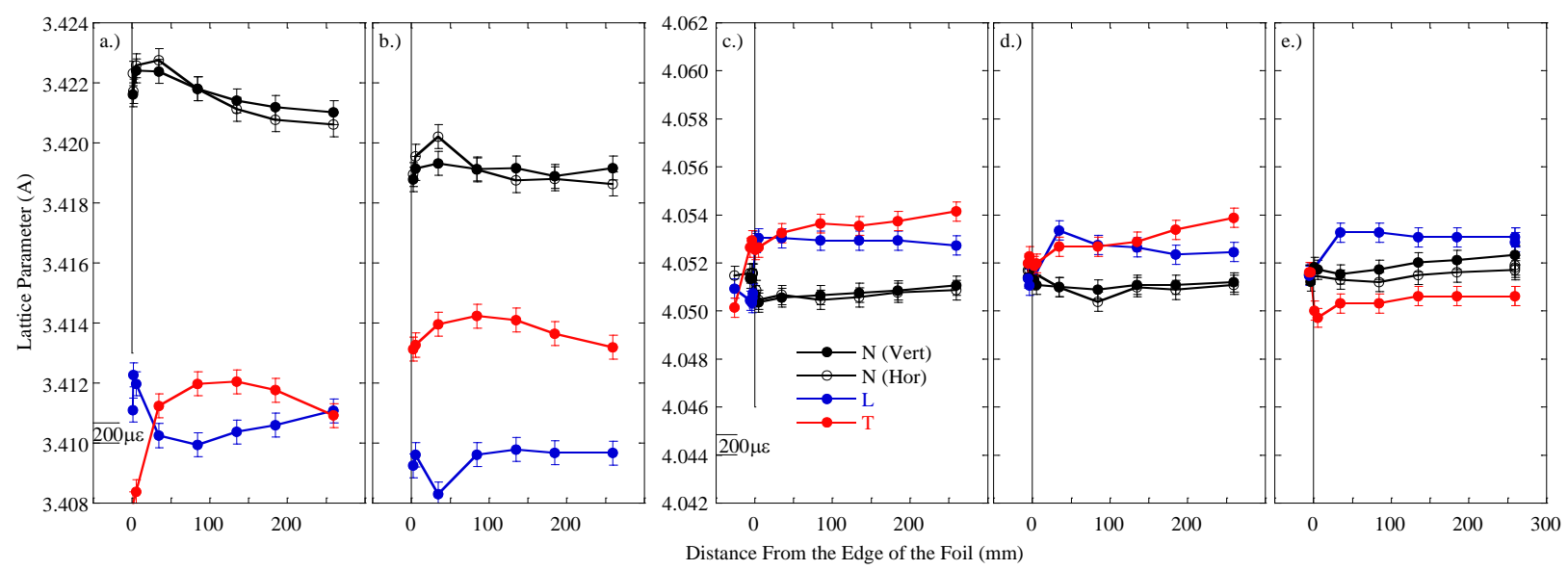

Figure 8 a,b.) U10Mo lattice parameters measured in the three sample directions in the OSU plate as a function of distance from the end of the plate on the a.) center line and b.) near the long edge of the fuel foil. c-e.) Same for Al lattice parameters measured on the c.) center line, d.) near the long edge of the fuel foil, and e.) $2 \mathrm{~mm}$ off the edge of the fuel foil. The open and closed black symbols represent the two independent measurements of the normal lattice parameter with the sample vertical and horizontal, respectively. The vertical black line at $40 \mathrm{~mm}$ represents the short edge of the fuel foil.

Figures 8a-e show similar plots (again on the same scales) of the lattice parameter determined on the OSU plate on similar lines, although more lines were measured on this sample. Recall that in the OSU plate, both measurements of the lattice parameter in the sample normal direction were completed in bank 1 . The directional dependent separation of the U-10Mo and $\mathrm{Al}$ lattice parameters is larger in the OSU plate than either mini-plate, corresponding to nearly $3000 \mu \varepsilon$ in the U-10Mo and $600 \mu \varepsilon$ in the Al.

Reported literature values of c/a for pure Zr range between 1.5926 and 1.5930 [30-32]. Table 1 enumerates the observed c/a ratios measured in a reference $\mathrm{Zr}$ foil and at the center of each of the mini-plates using the dominant grain orientations, that is $\mathbf{c}$ in the normal direction and $\mathbf{a}$ in either in-plane direction. The uncertainty in the reported c/a ratios is $\sim 0.0005$. The reference value agrees well with that found in the literature. In all cases, the c/a ratios in the clad samples are significantly larger than the literature and measured reference values, as much as $0.4 \%$ larger in the case of the OSU plate. In the as-HIP'ed mini-plates $\frac{c_{N}}{a_{T}}$ is consistently lower, 
that is closer to the equilibrium value, than $\frac{c_{N}}{a_{L}}$. Specifically, $\frac{c_{N}}{a_{L}}$ in the fast cooled mini-plate $(69-4)$ is $0.37 \%$ larger than the reference, while $c_{N} / a_{T}$ is $0.27 \%$ larger than the reference. Finally, the c/a ratios in the slow-cooled mini-plate are consistently lower than in the fast-cooled sample.

Following shearing of $69-4, \frac{c_{N}}{a_{T}}$ is larger than $\frac{c_{N}}{a_{L}}$.

\section{Discussion}

\subsection{Residual Stresses}

The directional dependence of the lattice parameter is a result of residual stresses presumably generated between the $\mathrm{Al}, \mathrm{Zr}$ and $\mathrm{U}-10 \mathrm{Mo}$ during cooling due to the difference in the coefficient of thermal expansion (CTE) between the three constituent materials. From examination of figures 5-7, it is clear qualitatively that the U-10Mo is either in in-plane compression or out-of-plane tension, resulting in the decreased lattice parameter in the sample in-plane directions. The thinness of the sample limits the out-of-plane stresses to be near zero, so we conclude that the U-10Mo must be in in-plane compression. The opposite sense of the directional dependence in the $\mathrm{Al}$ indicates that the stresses are balancing those in the $\mathrm{U}-10 \mathrm{Mo}$, that is the $\mathrm{Al}$ is in in-plane tension. Likewise, the offset of the observed c/a ratios from literature values suggest the $\mathrm{Zr}$ diffusion barrier is in in-plane compression. These results are to be expected based on the relative CTE's of the U-10Mo foil, the $\mathrm{Zr}$ diffusion barrier and the Al cladding. It is also evident, qualitatively, that the stresses are much reduced in the slow cooled sample and that removal of material (aluminum) through the shearing and thinning process on sample 69-4 forced redistribution of the residual stress field, resulting in the observed change in the stress. 

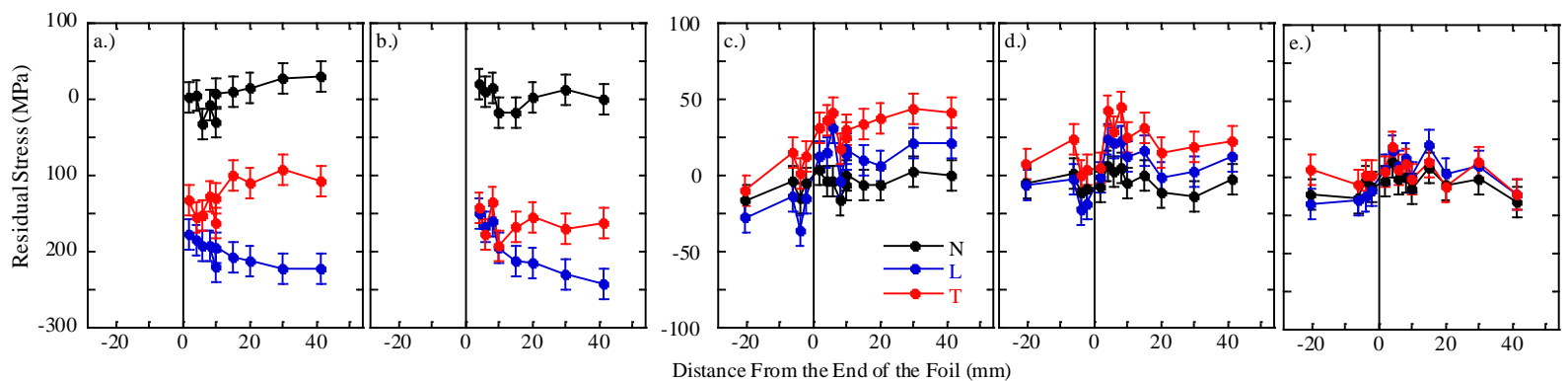

Figure 9 a,b.) Residual stresses measured in the U-10Mo foil in the three sample directions in the fast cooled sample, $69-4$, as a function of distance from the end of the fuel foil on the a.) center line and b.) $2 \mathrm{~mm}$ from the edge of the fuel foil.c-e.) Same for Al lattice parameters measured on the c.) center line, d.) $2 \mathrm{~mm}$ from the edge of the fuel foil, and e.) $2 \mathrm{~mm}$ off the edge of the fuel foil.

Figures 9a-e show the residual stresses in the U-10Mo fuel foil (a-b) and Al cladding (ce) in the fast cooled sample, 69-4, on the various line profiles; the scales are held fixed across the plots. In both the U-10Mo and $\mathrm{Al}$, the normal stress is everywhere within uncertainty of zero. Recall that we have assumed that to be the case only near the center of the foil. The predominant stress in the U-10Mo is in-plane compression to an absolute maximum of roughly -250MPa near the center of the foil in the longitudinal direction. Correspondingly, in the two line profiles over the U-10Mo, the stress in the aluminum is in-plane tension, but only as high as 50MPa over the center of the U-10Mo foil. All three stress components in the Al cladding reduce to within uncertainty of zero on the line profile $2 \mathrm{~mm}$ off the edge of the U-10Mo foil.
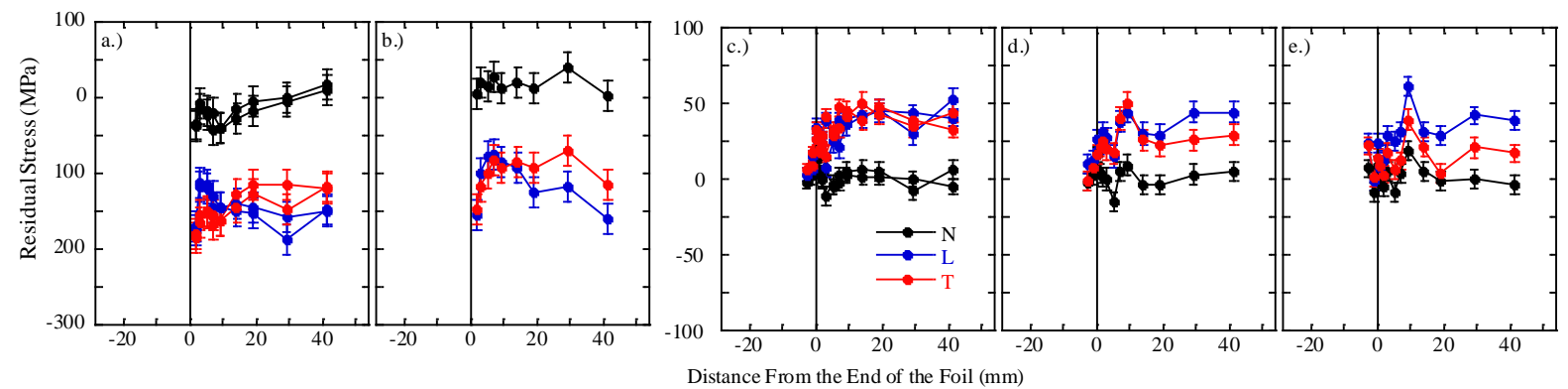

Figure 10 a,b.) Residual stresses measured in the U-10Mo foil in the three sample directions in the fast cooled sample, 69-4, following shearing and thinning to final dimensions as a function of distance from the end of the fuel foil on the a.) center line and b.) $2 \mathrm{~mm}$ from the edge of the fuel foil. c-e.) Same for Al lattice parameters measured on the c.) center line, d.) $2 \mathrm{~mm}$ from the edge of the fuel foil, and e.) $2 \mathrm{~mm}$ off the edge of the fuel foil. 
Figures 10a-e show the residual stresses in the same foil following shearing and thinning to final dimensions. The normal component of the residual stress has remained within uncertainty of zero, but the in-plane components evolve following the shearing operation. For instance, along the center line, the longitudinal stress decreased in magnitude from $-250 \mathrm{MPa}$ to roughly $-175 \mathrm{MPa}$. Near the edge of the foil, both in-plane components decrease in magnitude, from near -200MPa, to roughly -100MPa.
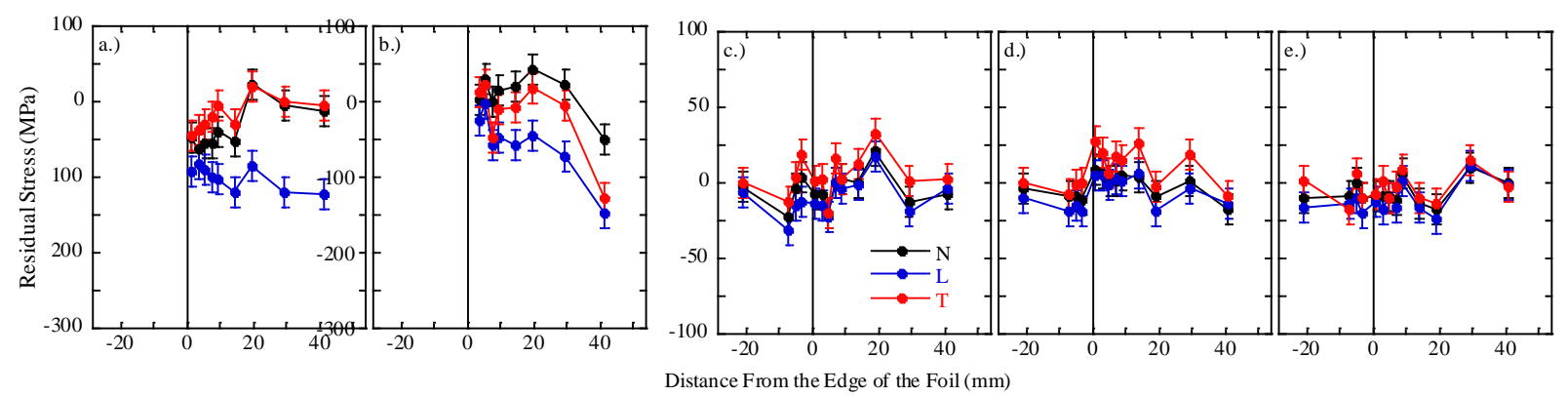

Figure 11 a,b.) Residual stresses measured in the U-10Mo foil in the three sample directions in the slow cooled sample, $68-5$, as a function of distance from the end of the fuel foil on the a.) center line and b.) $2 \mathrm{~mm}$ from the edge of the fuel foil. c-e.) Same for Al lattice parameters measured on the c.) center line, d.) $2 \mathrm{~mm}$ from the edge of the fuel foil, and e.) $2 \mathrm{~mm}$ off the edge of the fuel foil.

Figures 11a-e show the residual stresses in the U-10Mo fuel foil (a-b) and Al cladding (ce) in the slow cooled sample, 68-5, on the various line profiles. The scales are held fixed with respect to those in figures 8 and 9. The stresses in the U-10Mo are considerably reduced in the slow cooled sample, roughly $-100 \mathrm{MPa}$ in the longitudinal direction at the center of the foil, and near zero in the transverse and normal directions. In the U-10Mo near the foil edge the residual stress ranges from 0 to $-100 \mathrm{MPa}$ for the in plane stresses and from 0 to $-50 \mathrm{MPa}$ for the out of plane stress. In the Al cladding, the stresses are within uncertainty of zero.

Figures $12 \mathrm{a}$ and $\mathrm{b}$ show residual stress profiles for both the aluminum cladding and U$10 \mathrm{Mo}$ foil in the OSU plate over a quarter of the plate. The scale for the aluminum profiles ranges from $-80 \mathrm{MPa}$ to $70 \mathrm{MPa}$ in $10 \mathrm{MPa}$ increments, while that of the $\mathrm{U}-10 \mathrm{Mo}$ profile ranges 
from $-325 \mathrm{MPa}$ to $100 \mathrm{MPa}$ in $25 \mathrm{Mpa}$ intervals. The $*$ on the figure indicate the actual diffraction positions. The white * indicate positions where the $\mathrm{Al}$ diffraction pattern was affected by neutrons scattered from the $\mathrm{Al}$ clamps (see figure 2c) and the strains in the $\mathrm{Al}$ have been interpolated at these points in order to have a uniform grid for the contour plot.

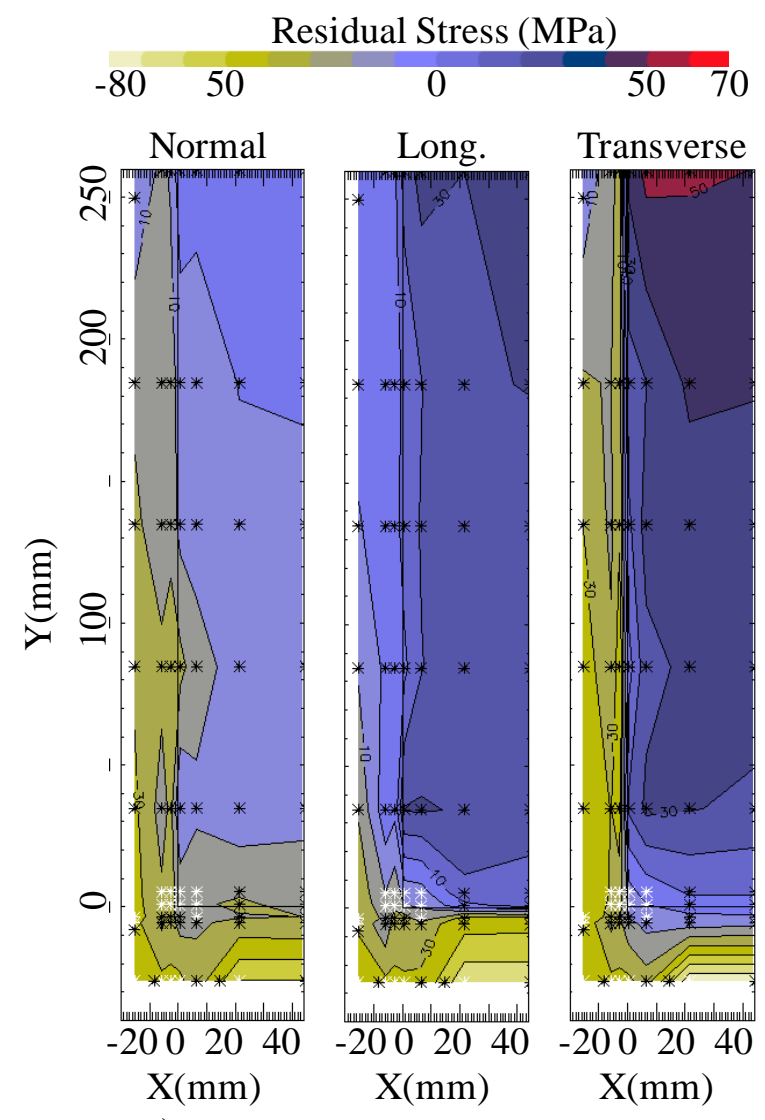

a.)

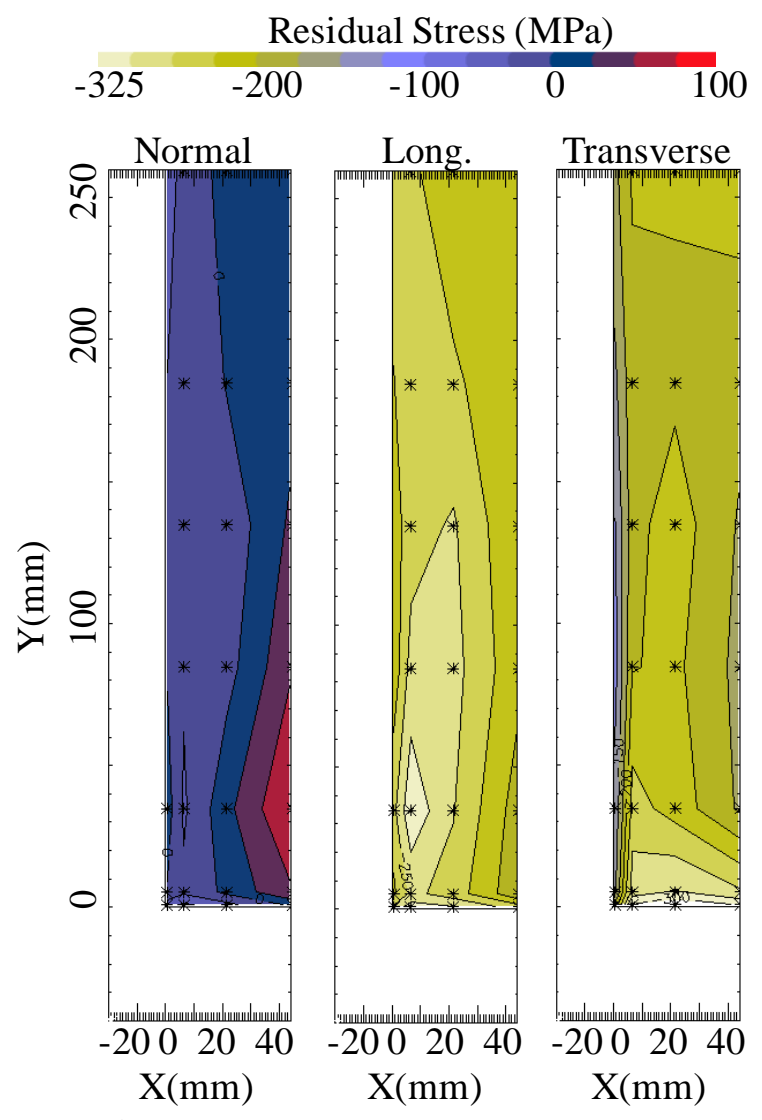

b.)

Figure 12. Residual stress profiles in the (a.)Al cladding and (b.) U10Mo fuel foil in the OSU plate.

The normal stresses in both phases were assumed to be zero near the center of the foil, i.e. at $(\mathrm{x}, \mathrm{y})=(63.7 \mathrm{~mm}, 300 \mathrm{~mm})$. The normal stresses differ little from zero, except near the edges. The in-plane stress components are more significant. The longitudinal stress in the aluminum is roughly $30 \mathrm{MPa}$ everywhere over the fuel foil. It drops very quickly to zero and becomes negative when crossing the short edge, i.e. when the stress component is normal to the 
edge. In contrast, the longitudinal stress component drops more slowly when crossing the long edge, that is when the stress component is parallel to the boundary. The transverse stress is similar, approaching 50MPa near the center of the foil. Similar to the behavior of the longitudinal stress, when crossing the edge that is normal to the stress component, in this case the long edge, the transverse stress decreases rapidly to zero or even slightly compressive. In contrast to the longitudinal stress, the transverse stress also decreases relatively quickly when crossing the short edge, when the stress component is parallel to the boundary. The difference in the behavior is likely due to the fact that the edge effects will be more dramatic on the short side of the foil.

The in-plane, longitudinal and transverse stresses in the U-10Mo are similar and compressive, roughly -200MPa near the center of the foil and reaching an absolute maximum at roughly $-275 \mathrm{MPa}$ closer to the corner. The magnitude of the stress field is similar to that in the mini-plates, but the trends are somewhat different, especially near the edges of the fuel foil [Brown, 2013 \#1887].

\subsection{Comparison of Neutron and X-ray Measurements}

Figure 13a-b shows the stresses in the U-10Mo foil of sample 69-4 determined with neutron diffraction in this work compared to high energy x-ray diffraction measurements that were previously completed at the APS [5]. On the centerline, the results are generally within uncertainty, although the longitudinal stresses determined with neutron diffraction are consistently more compressive than those determined with x-rays. At $2 \mathrm{~mm}$ from the edge of the sample, the sets of results show the same trends, but the neutron diffraction results are consistently $\sim 50 \mathrm{MPa}$ more compressive then those determined with x-rays. 


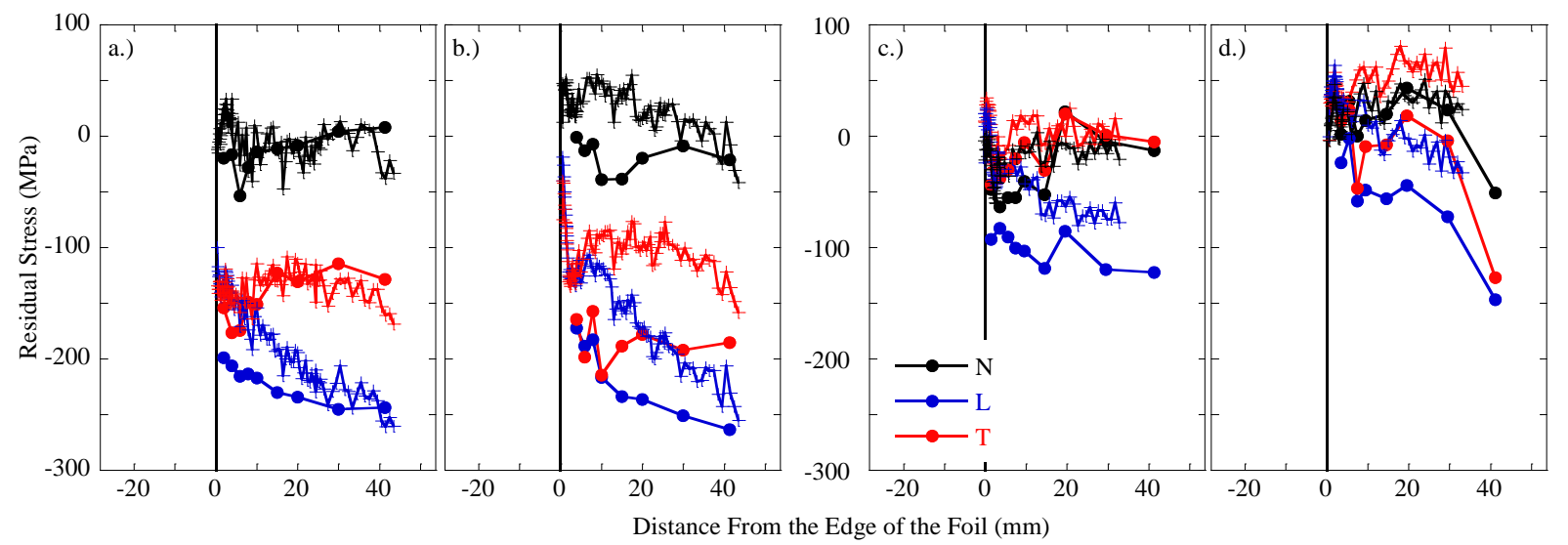

Figure 13 Comparison of the residual stresses in U-10Mo foil determined at beamline 1ID at the APS (+'s) and SMARTS (•'s) at the Lujan Center on line profiles on mini-plate 69-4 at the a.) center line and b.) $2 \mathrm{~mm}$ from the edge and mini-plate 68.5 at the c.) center line and d.) $2 \mathrm{~mm}$ from the edge.

It is difficult to immediately make a value judgment between the two measurements. There has always been the issue of calibration and reference lattice parameter measurements, which has been discussed in each publication [5]. In this case, it appears the measurements agree in one position, the center line, and not in another, the edge, which suggests that the calibration changed with position in the sample. Given the on-board calibration, it is difficult to see how this could occur in the neutron diffraction measurement. It is possible, despite the rigorous efforts to align the sample during the $\mathrm{x}$-ray measurements, that the sample motion was not normal to the beam if the sample axes were not perfectly aligned with the translation state. Another way to look at this is that the measurements accurately determined the deviatoric stresses, but struggle to determine the hydrostatic stress components due to calibration and reference issues.

\subsection{Zr Diffusion Barrier}

Solving for the strain and substituting into the above equations, the literature value of $\frac{c_{0}}{a_{0}}=1.5928$, and appropriate elastic properties for $\mathrm{Zr}(\mathrm{E}=100 \mathrm{GPa}$ and $\mathrm{n}=0.3$ [33]) the in-plane component of the residual stress in the $\mathrm{Zr}$ is estimated to approach $-300 \mathrm{MPa}$ in the case of the 
OSU plate. Note, this estimation assumes isotropic elastic properties of $\mathrm{Zr}$ [33], which is not a good approximation, but is sufficient to get an estimate of the residual stress in the diffusion barrier layer. The magnitude of the stress is large, but sensible. Table 1 shows estimated stresses for the diffusion barrier in each sample and each in-plane direction. Certainly the estimate described above does not justify three significant digits in the reporting of the absolute stresses in the $\mathrm{Zr}$ diffusion barrier, but in a relative sense, it is useful when comparing stresses calculated in the same manner.

\section{Conclusion}

Residual stresses were measured in two Al-clad U-10Mo mini-plates, which were subjected to a fast-cooling and slow-cooling procedure from the cladding bonding process (hot isostatic pressing) and a full size plate, called the OSU plate. The fast-cooled mini-plate was measured again following the shearing and thinning of the Al cladding to the final dimensions, which are required for reactor testing. Stresses were determined quantitatively in the Al cladding and U10Mo fuel foil, and estimated in the $\mathrm{Zr}$ diffusion barrier layer. The observed stresses in the U10Mo foil of the fast cooled mini-plate agree in trend with those determined with high energy $\mathrm{x}$ rays, but show an offset with respect to previous high-energy x-ray measurements [5].

In the U-10Mo foil, the stresses are predominantly in-plane compression. They were larger in the fast-cooled mini-plate, reaching $-250 \mathrm{MPa}$ in the longitudinal direction, and only $100 \mathrm{MPa}$ in the slow cooled mini-plate. This demonstrates that the slow cooling rates, which are more typical for commercial sized HIPs can help to reduce the residual stresses in the fuel plates. In-plane tensile stresses are observed in the aluminum in the fast-cooled mini-plate, reaching up to $\sim 50 \mathrm{MPa}$, but in the slow-cooled mini-plate, the stresses are reduced to the level of the 
measurement uncertainty. The in-plane stresses in the $\mathrm{Zr}$ diffusion barrier layer at the center of the plates were estimated to approach roughly $-300 \mathrm{MPa}$ in the mini-plates.

Shearing and thinning the Al cladding to final dimensions causes a significant evolution of the stresses in both the U-10Mo fuel foil and Al cladding. The in-plane stresses in the fuel foil were reduced with respect to the as-HIP'ed sample. Detailed finite element analysis should be able to predict this response. It is interesting to note that the reference lattice parameter that was determined by forcing the normal stress to zero in the center of the plate was repeatable between the two measurements in mini-plate $69-4$, separated by several months.

The in-plane stresses were largest in the full sized OSU fuel plate, reaching $-275 \mathrm{MPa}$ in the U-10Mo and greater that 50MPa in the aluminum. The trends are somewhat different in the larger plate, with the maxima in the stress near the corner of the fuel foil, rather than in the center as is the case for the mini-foils.

\section{Acknowledgements}

This research was supported by the U.S. Department of Energy (DOE) and the National Nuclear Security Administration's Office of Materials Management and Minimization through the DOE Idaho Operations Office Contract DE-AC07-05ID14517. This work has benefited from the use of the Manual Lujan, Jr. Neutron Scattering Center at LANSCE, which was funded by the United States Department of Energy's Office of Basic Energy Sciences. Los Alamos National Laboratory is operated by Los Alamos National Security LLC under Department of Energy contract DE-AC52-06NA25396. 


\section{References}

[1] J.L. Snelgrove, G.L. Hofman, M.K. Meyer, C.L. Trybus, T.C. Wiencek, Nuclear Engineering and Design, 187 (1997) 119-126.

[2] J.F. Jue, B.H. Park, C.R. Clark, G.A. Moore, D.D. Keiser, Nuc Tech, 172 (2010) 204-210.

[3] E. Perez, B. Yao, D.D. Keiser, Jr., Y.H. Sohn, J Nuc Matl, 402 (2010) 8-14.

[4] S. Vogel, D.W. Brown, M.A. Okuniewski, Los Alamos National Lab LAUR-10-05912, 2010, Los Alamos, NM

[5] D.W. Brown, M.A. Okuniewski, J.D. Almer, L. Balogh, B. Clausen, J.S. Okasinski, B.H. Rabin, J Nuc Matl, 441 (2013) 252-261.

[6] A.D. Krawitz, Mater. Sci. Technol., 27 (2011) 589-603.

[7] M.T. Hutchings, P.J. Withers, T.M. Holden, T. Lorentzen, Introduction to the Characterization of Residual Stress by Neutron Diffraction, Routledge, USA, 2005.

[8] M.A.M. Bourke, D.C. Dunand, E. Ustundag, App. Phys. A, A74 (2002) S1707-S1709.

[9] X.L. Wang, T.M. Holden, G.Q. Rennich, A.D. Stoica, P.K. Liaw, H. Choo, C.R. Hubbard, Physica B, 385 (2006) 673-675.

[10] S. Harjo, K. Aizawa, T. Ito, H. Arima, J. Abe, A. Moriai, K. Sakasai, T. Nakamura, T. Nakatani, T. Iwahashi, T. Kamiyama,in: Y. Akiniwa, K. Akita, H. Suzuki (Eds.) Mechanical Stress Evaluation by Neutrons and Synchrotron Radiation, Trans Tech Publications Ltd, StafaZurich, 2010, pp. 99-104.

[11] J.R. Santisteban, M.R. Daymond, J.A. James, L. Edwards, J. App. Crys., 39 (2006) 812-825.

[12] D.F. Sears, N. Wang, R. Rogge, I.P. Swainson, R. Donaberger,in RRFM 2012

Transactions, European Research Reactor Conference, Prague, Czech Republic, 2012, pp. 45-50.

[13] M.A.M. Bourke, J.A. Goldstone, T.M. Holden,in: M.T. Hutchings, A.D. Krawitz (Eds.) Measurement of Residual and Applied Stress Using Neutron Diffraction, Kluwer Academic Publishers, The Netherlands, 1992, pp. 369-382.

[14] D.W. Brown, T.M. Holden, B. Clausen, M.B. Prime, T.A. Sisneros, H. Swenson, J. Vaja, Acta. Mat., 59 (2011) 864-873.

[15] D.Q. Wang, X.L. Wang, J.L. Robertson, C.R. Hubbard, J. App. Crys., 33 (2000) 334-337.

[16] X.L. Wang, Y.D. Wang, J.W. Richardson, J. App. Crys., 35 (2002) 533-537.

[17] H. Ozaltun, M.H.H. Shen, P. Medvedev, J Nuc Matl, 419 (2011) 76-84. 
[18] R.B. Vondreele, J.D. Jorgensen, C.G. Windsor, J. App. Crys., 15 (1982) 581-589.

[19] A.C. Larson, R.B. Von Dreele, Los Alamos National Lab 1986, Los Alamos, NM

[20] C.N. Tupper, D.W. Brown, R.D. Field, T.A. Sisneros, B. Clausen, Met. Trans. A, 43A (2012) 520-530.

[21] R.B. Von Dreele, J. App. Crys., 30 (1997) 517-525.

[22] B. Clausen, Los Alamos National Lab LA-UR 04-6581, 2004, Los Alamos, NM

[23] J.M. Gere, S.P. Timoshenko, Mechanics of Materials, PWS Publishing Company, 1997.

[24] I.C. Noyan, J.B. Cohen, Residual Stress-Measurement by Diffraction and Interpretation, Springer-Verlag, New York., 1987.

[25] J.E. Gates, E.G. Bodine, J.C. Bell, A.A. Bauer, G.D. Calkins, Batelle Memorial Institute BMI-APDA-638, 1958, Columbus, Oh

[26] A.M. Nomine, D. Bedere, D. Miannay,in J.J. Burke, D.A. Colling, A.E. Gorum, J. Greenspan (Eds.) Physical Metallugy of Uranium Alloys :Third Army Materials Technology Conference, Brook Hill Publishing Company, Vail, Colorado, 1974, pp. 657-700.

[27] D.W. Brown, D.J. Alexander, K.D. Clarke, B. Clausen, M.A. Okuniewski, T.A. Sisneros, Scripta Mat., 69 (2013) 666-669.

[28] S.R. MacEwen, C. Tomé, J. Faber, Acta. Met., 37 (1989) 979-989.

[29] D.W. Brown, T.A. Sisneros, B. Clausen, S. Abeln, M.A.M. Bourke, B.G. Smith, M.L. Steinzig, C.N. Tome, S.C. Vogel, Acta. Mat., 57 (2009) 972-979.

[30] H.E. Swanson, R.K. Fuyat, National Bureau of Standards (U.S.), 539 (1953) 1-65.

[31] J. Goldak, L.T. Lloyd, C.S. Barrett, Physical Review, 144 (1966) 478-484.

[32] R.B. Russell, JOM, 6 (1954) 1045-1052.

[33] D. Tromans, International Journal of Research and Reviews in Applied Sciences, 6 (2011) 462-483.

[34] M.E. Straumanis, L.S. Yu, Acta Crystallographica Section A-Crystal Physics Diffraction Theoretical and General Crystallography, A 25 (1969) 676. 
Table 1

\begin{tabular}{|c|c|c|c|c|c|c|c|c|c|}
\hline Literature & $\begin{array}{c}\text { Rolled } \\
\mathrm{Zr}\end{array}$ & \multicolumn{2}{|c|}{$69-4$} & \multicolumn{2}{c|}{$69-4$ sheared } & \multicolumn{3}{c|}{$68-5$} & \multicolumn{3}{c|}{ OSU } \\
\hline $\mathrm{c} / \mathrm{a}$ & $\mathrm{c}_{\mathrm{N}} / \mathrm{a}_{\mathrm{T}}$ & $\mathrm{c}_{\mathrm{N}} / \mathrm{a}_{\mathrm{L}}$ & $\mathrm{c}_{\mathrm{N}} / \mathrm{a}_{\mathrm{T}}$ & $\mathrm{c}_{\mathrm{N}} / \mathrm{a}_{\mathrm{L}}$ & $\mathrm{c}_{\mathrm{N}} / \mathrm{a}_{\mathrm{T}}$ & $\mathrm{c}_{\mathrm{N}} / \mathrm{a}_{\mathrm{L}}$ & $\mathrm{c}_{\mathrm{N}} / \mathrm{a}_{\mathrm{T}}$ & $\mathrm{c}_{\mathrm{N}} / \mathrm{a}_{\mathrm{L}}$ & $\mathrm{c}_{\mathrm{N}} / \mathrm{a}_{\mathrm{T}}$ \\
\hline 1.5928 & 1.5929 & 1.5987 & 1.5971 & 1.5963 & 1.5971 & 1.5982 & 1.5965 & 1.5991 & 1.5990 \\
\hline Estimated & & -280 & -210 & -170 & -210 & -260 & -180 & -300 & -300 \\
in-plane & & $(\mathrm{L})$ & $(\mathrm{T})$ & $(\mathrm{L})$ & $(\mathrm{T})$ & $(\mathrm{L})$ & $(\mathrm{T})$ & $(\mathrm{L})$ & $(\mathrm{T})$ \\
stress $(\mathrm{MPa})$ & & & & & & & & & \\
\hline
\end{tabular}

Table 1. c/a ratio in reference $\mathrm{Zr}$ and the studied plates. 


\section{Figure Captions}

Figure 1. Schematic of the (a.) mini-plate and (b.) OSU samples with an overlaid plot of the measurement positions. The origin of the axis used throughout this report is as shown. The longitudinal (2) and transverse (1) directions are indicated. The normal (3) direction is out of the page.

Figure 2. a.) Schematic of the diffraction geometry of SMARTS indicating the diffraction vectors of each detector bank. Pictures of the b.) the mini plates and c.) OSU plate in SMARTS. The samples are shown as mounted to determine the lattice parameters in the $\mathrm{T}$ and $\mathrm{N}$ sample directions of the mini plates and $\mathrm{N}$ and L sample directions of the OSU plate in banks 1 and 2, respectively.

Figure 3. Inverse pole figures representing the texture of the U10 Mo in the (a.) fast cooled (694), (b.) slow cooled (68-5) and (c.) OSU plates. Contours go from 0 to 4 multiples of random distribution (MRD) at 0.25 intervals.

Figure 4. a.) Raw and corrected $\mathrm{Cu}$ and $\mathrm{Zr}$ b.) transverse and c.) normal lattice parameters as a function of distance offset from the diffractometer center.

Figure 5 a,b.) U10Mo lattice parameters measured in the three sample directions in the fast cooled sample, 69-4, as a function of distance from the end of the foil on the a.) center line and b.) $2 \mathrm{~mm}$ from the edge of the fuel foil. c-e.) Same for Al lattice parameters measured on the c.) center line, d.) $2 \mathrm{~mm}$ from the edge of the fuel foil, and e.) $2 \mathrm{~mm}$ off the edge of the fuel foil. The open and closed black symbols represent the two independent measurements of the normal lattice parameter with the sample vertical and horizontal, respectively. The vertical black line at $0 \mathrm{~mm}$ represents the edge of the fuel foil.

Figure 6 a,b.) U10Mo lattice parameters measured in the three sample directions in the fast cooled sample, 69-4, following shearing and thinning to final dimensions as a function of distance from the end of the foil on the a.) center line and b.) $2 \mathrm{~mm}$ from the edge of the fuel foil. c-e.) Same for Al lattice parameters measured on the c.) center line, d.) $2 \mathrm{~mm}$ from the edge of the fuel foil, and e.) $2 \mathrm{~mm}$ off the edge of the fuel foil.

Figure 7 a,b.) U10Mo lattice parameters measured in the three sample directions in the slow cooled sample, 68-5, as a function of distance from the end of the foil on the a.) center line and b.) $2 \mathrm{~mm}$ from the edge of the fuel foil. c-e.) Same for Al lattice parameters measured on the c.) center line, d.) $2 \mathrm{~mm}$ from the edge of the fuel foil, and e.) $2 \mathrm{~mm}$ off the edge of the fuel foil.

Figure 8 a,b.) U10Mo lattice parameters measured in the three sample directions in the OSU plate as a function of distance from the end of the plate on the a.) center line and b.) near the long edge of the fuel foil. c-e.) Same for Al lattice parameters measured on the c.) center line, d.) near the long edge of the fuel foil, and e.) $2 \mathrm{~mm}$ off the edge of the fuel foil. The open and closed 
black symbols represent the two independent measurements of the normal lattice parameter with the sample vertical and horizontal, respectively. The vertical black line at $40 \mathrm{~mm}$ represents the short edge of the fuel foil.

Figure 9 a,b.) Residual stresses measured in the U-10Mo foil in the three sample directions in the fast cooled sample, 69-4, as a function of distance from the end of the fuel foil on the a.) center line and b.) $2 \mathrm{~mm}$ from the edge of the fuel foil. c-e.) Same for Al lattice parameters measured on the c.) center line, d.) $2 \mathrm{~mm}$ from the edge of the fuel foil, and e.) $2 \mathrm{~mm}$ off the edge of the fuel foil.

Figure 10 a,b.) Residual stresses measured in the U-10Mo foil in the three sample directions in the fast cooled sample, 69-4, following shearing and thinning to final dimensions as a function of distance from the end of the fuel foil on the a.) center line and b.) $2 \mathrm{~mm}$ from the edge of the fuel foil. c-e.) Same for Al lattice parameters measured on the c.) center line, d.) $2 \mathrm{~mm}$ from the edge of the fuel foil, and e.) $2 \mathrm{~mm}$ off the edge of the fuel foil.

Figure 11 a,b.) Residual stresses measured in the U-10Mo foil in the three sample directions in the slow cooled sample, 68-5, as a function of distance from the end of the fuel foil on the a.) center line and b.) $2 \mathrm{~mm}$ from the edge of the fuel foil. c-e.) Same for Al lattice parameters measured on the c.) center line, d.) $2 \mathrm{~mm}$ from the edge of the fuel foil, and e.) $2 \mathrm{~mm}$ off the edge of the fuel foil.

Figure 12. Residual stress profiles in the (a.)Al cladding and (b.) U10Mo fuel foil in the OSU plate.

Figure 13 Comparison of the residual stresses in U-10Mo foil determined at beamline 1ID at the APS (+'s) and SMARTS ( $\cdot$ 's) at the Lujan Center on line profiles on mini-plate 69-4 at the a.) center line and b.) $2 \mathrm{~mm}$ from the edge and mini-plate 68.5 at the c.) center line and d.) $2 \mathrm{~mm}$ from the edge. 